\title{
Dynamic spectral characteristics of defective sounds pronunciation in students with minimal dysarthric disorders
}

\author{
Ludmila Lopatina ${ }^{1}$, Ludmila Baryaeva $^{2}$, and Mariya Ivleva $^{2 *}$ \\ ${ }^{1}$ Herzen State Pedagogical University of Russia, 191186, Saint-Petersburg, Russia \\ ${ }^{2}$ Moscow City University, 129226, Moscow, Russia
}

\begin{abstract}
The article is devoted to the analysis of the spectrographic characteristics of defective sounds pronunciation in children with minimal dysarthric disorders (mild dysarthria). These characteristics were obtained in the process of an experimental-phonetic study. It involved the study of the nature of changes that occur during the transition from one sound to another and also during the pronunciation of a single sound. The article presents the results of the experiment carried out on the basis of a qualitative analysis of dynamic spectrograms of syllables; describes the most permanent signs characterizing the defective sounds pronunciation in syllables of various structures (open, closed). The nature of the mutual influences of sounds in various structures of syllables is revealed. Comparative data were obtained for the acoustic (formant) proximity of various variants of defective pronunciation to the sound standard in children with minimal dysarthric disorders. The contextual phonetic influences at the level of syllables are determined, taking into account the acoustic characteristics of sounds and their defective variants.
\end{abstract}

\section{Introduction}

Pronunciation defects, which are associated with specific motor disorders in speech realization, are widely and variably presented in the structure of a speech defect in children with minimal dysarthric disorders (mild dysarthria). They arise as a result of damage to various brain structures involved in the control of the motor mechanism of speech [1-4].

Disorders of motor regulation of articulatory organs lead to changes in the acoustic characteristics of speech [5-7]. The articulatory positions of speech sounds determine the originality of their acoustic characteristics. Any deviation, even insignificant, in the position and work of the articulatory organs causes a dynamic change in the acoustic image of sound [8-10].

Sound units are complex in their acoustic nature. They have such characteristics as pitch, strength, timbre, duration of sound. The most essential feature of sound units is their inherent sound spectrum, characterized by the presence of a number of components (formants) in certain frequency fields. These formants have an increased intensity (in

\footnotetext{
* Corresponding author: m.g.ivleva@yandex.ru
} 
comparison with other components). The classical position of experimental phonetics is that the frequency of F1 is associated with such a sign of a vowel as its rise (or the degree of openness - closeness). The frequency of F2 is associated with a sign of a row (i.e., with the degree of forward or backward movement). And the frequency F3 reacts to a change in the row sign only at a high value of the F2 frequency, i.e. when varying non-posterior vowels. Formant trajectory on the transition areas correspond to those articulatory rearrangements that occur when articulation changes from consonant to vowel or from vowel to consonant $[11,12]$.

Formants inherent in each phoneme are its objective features, which make it possible to recognize phonemes and differentiate them in the process of speech perception.

The fulfillment of these features (mainly, the formant) is an indispensable condition for intelligibility of speech. And vice versa, illegibility, slurred speech occurs due to a disorder of the acoustic features of phonemes, which lead to distortion of speech sounds. This manifests itself especially clearly when, for some reason, the formants inherent in these or other phonemes are absent or weakly expressed in speech.

There are complex relationships between phonemes and the spectral characteristics of their corresponding signals. Not only defects in sound pronunciation act as factors influencing the nature of the speech signal (its variation). The implementation of this or that option in a specific act of communication depends on solely linguistic reasons, primarily on the environment of the varying form, i.e. from the context. Its influence causes the shift of the signal energy to different frequency ranges, the formation of transitional segments that characterize both sounds simultaneously [13].

In this regard, it can be claimed that specific defects in articulation at minimal dysarthric disorders lead to the appearance of qualitatively different changes in the spectral characteristics of sounds [14-16]. Currently, in Russian speech therapy, the linguistic aspect of sound-pronunciation disorders in children with this type of speech dysontogenesis is practically not developed. At the same time, the usage of data, which were obtained using instrumental methods (spectrography), will help to understand the pathogenesis of the pronunciation defect in minimal dysarthric disorders (mild dysarthria), to determine the selection criteria for the linguistic material that can be used in speech therapy work [17, $18]$.

\section{Methods and materials}

The study of the spectral characteristics of sounds was carried out on the material of the phonetic group of hissing sounds, which are most often disturbed in pronunciation (along with sibilant and sonorous sounds). A voiceless, hard consonant [sh] was selected from the group of hissing sounds for the experiment. Syllables of various structures were selected: CV (consonant - vowel) and VC (vowel - consonant) with this sound. Open and closed syllables were a combination of a voiceless slit consonant [sh] with different vowels: [a], $[\mathrm{o}],[\mathrm{u}],[\mathrm{y}]$. The choice of the sound [sh] from the group of hissing sounds was due to the fact that the correct pronunciation of this sound is a necessary condition for correcting the defective pronunciation of other hissing sounds.

Research data were obtained on a probabilistic sample of two groups of children (experimental group - children with minimal dysarthric disorders and a group for comparative analysis - children without speech disorders). The age of children was 5.5-6.5 years. Children who could read were presented with syllables written on cards, which they were asked to read. The syllables spoken by the children were recorded in the audio recording mode. The recording was carried out in a studio with soundproofing through a studio microphone at a speed of $19 \mathrm{~mm} / \mathrm{s}$ with an interval between syllables of 5-7 seconds. Children with defective pronunciation of the sound [sh] (interdental, dental, 
lateral, labiodental, labial / buccal) were the announcers. For comparison, the normative pronunciation of this sound in various syllable structures was recorded as a reference standard. The audio recordings were processed using a computer in order to obtain dynamic spectrograms illustrating the frequency characteristics of the recorded sounds that form syllables with vowels of different structure. Dynamic spectrograms were compared with those (with dynamic spectrograms), compiled by us earlier on the material of a voiceless, solid sound [s].

\section{Results}

Different features were analyzed in all cases when comparing the correct and impaired pronunciation of the slit voiceless sound [sh] in syllables of various structures. These features are the position of the upper and lower noise boundaries, the noise bandwidth, the formant frequency, the presence of areas of maximum energy concentration, compactness and diffuseness, noise intensity (high-intensity (sharp) and low-intensity (unsharp)).

Analysis of syllables spectrograms (the CV type) shows that in about $60 \%$ of cases, a decrease in the upper noise limit was observed. This feature was most consistently traced with the vowel [o]. In contrast to the upper border of noise, the decreasing of the lower border was found almost everywhere, in combination with all vowels for all types of defective pronunciation. The only exception was the syllable [shi] in labiodental sigmatism. Almost everywhere, an increase in the width of the noise bandwidth was also a stable sign, with the exception of the syllable [sho] in case of dental sigmatism, in this case it is wider with distorted pronunciation than with normative pronunciation to a greater or lesser extent. A significant increase in the width of the noise bandwidth with any vowels was observed with interdental, labiodental and labial (buccal) sigmatisms. For instance, the width of the noise bandwidth in the syllable [shu] is normally about $6.825 \mathrm{~Hz}$, and with labiodental, interdental and labial sigmatisms $-7.325,8.325 \mathrm{~Hz}$ and $8.750 \mathrm{~Hz}$, correspondingly.

It was found that all types of defective pronunciation, except for the lateral and labial ones, are characterized by the presence of a lower intensity of noise. This effect made possible to characterize the sound [sh] in interdental, labiodental and dental sigmatisms as unsharp, and as sharp in lateral and labial sigmatisms. There was a tendency to a decrease in the number of areas of maximum energy concentration, and these areas themselves (if any) were much narrower than during normal pronunciation, although they were located in the high-frequency range, as in the norm. In a number of cases, areas of maximum energy concentration were absent and the number of acoustic zeros in the spectrum increased (for example, with all types of defective pronunciation in combination with the vowel [a]; with labiodental and labial sigmatisms in combination with vowels $[\mathrm{u}]$ and $[y]$ ).

An increase in the number of formants was the most constant sign only in syllables in combination with the vowel [a], with all variants of defective pronunciation. In cases with other vowels, the amount of formants increased only with interdental and labial pronunciation. The sign of compactness manifested itself less often than the sign of diffuseness during impaired pronunciation. Generally the slit voiceless sound [sh] was diffuse in all types of defective pronunciation.

Thus, the most constant signs characterizing defective pronunciation of the sound [sh] in the structure of the syllable type CV were identified. The following signs were noted: lower border of the noise band; increasing the noise bandwidth; less noise intensity; tendency to an increase in the amount of formants. The last two features make it possible to speak about the lack of sharpness of sound in almost all defective pronunciation variants, except for the lateral and labial ones. The sign of diffuseness can be called relatively constant. 
The signs noted in the case of impaired pronunciation of the sound [sh] in the structure of a syllable of the CV type were observed as the most constant and with defects in pronunciation of the sound [s] in the structure of a similar syllable.

Analysis of the spectrograms of syllables such as VC shows that a constant sign for the dental pronunciation (in combination with all vowels) is a decrease in the upper border of the noise. This feature was optional in the case of lateral sigmatism. It was noted exclusively when combined with the vowel [a].

The decrease in the lower border of the noise bandwidth was quite constant (with the exception of only two cases: lateral and labial pronunciation of the sound [sh] in combination with the vowel $[\mathrm{u}]$ ). An increase in noise bandwidth was observed practically in all variants of impaired pronunciation (with the exception of dental sigmatism). Moreover, this was typical for syllables of the CV type to a greater extent with interdental, labiodental and labial pronunciation in combination with all vowels. Impaired pronunciation was characterized mainly by an increase in the intensity of noise and did not have a pronounced tendency to a decrease in the number of areas of maximum energy concentration (except for dental sigmatism, where these areas were absent, and areas of acoustic zeroes occupied a significant place). An increase in the number of formants was a more permanent feature in comparison with the previous structure of syllables.

The most constant sign was an increase in the number of formants only in combination with the vowel [a] for all types of distorted pronunciation in the case of pronouncing syllables of the CV type. Such an increase was also observed In syllables of the VC type in combination with other vowels and was found not only with interdental, but also with dental, labiodental and labial sigmatisms. As well as in syllables of the CV type, the sign of compactness manifested itself less frequently than the sign of diffuseness in all variants of defective pronunciation.

Thus, the most permanent signs were identified, they characterize the defective pronunciation of the sound [sh] in the structure of a syllable of the VC type. They include reducing the lower border of the noise bandwidth; increasing the noise bandwidth; increasing in the amount of formants; diffuseness; increasing noise intensity and maintaining areas of maximum energy concentration. The indicated signs, with the exception of the final one, were also observed in syllables of the CV type. Identical, most constant signs were noted that characterize the defective pronunciation of these sounds in the structure of syllables with the sound [sh], as well as in the structure of syllables such as $\mathrm{CV}$ with the sound [s].

The results of the study indicate that voiceless slit consonants [s] and [sh] in all types of distorted pronunciation are formed with the participation of a turbulent noise source due to the presence of narrowing in some place of the articulatory tract, which creates vortex flows that cause specific noise). These sounds are characterized by more specific frequency areas than explosive sounds, as in normative pronunciation. The voiceless slit [sh] is highly intense (especially when labial pronunciation is combined with all vowels), it has the widest band of noise. When pronouncing a voiceless slit sound [s] (in almost all types of distortions), the noise bandwidth is smaller compared to the sound [sh], but the own frequency of the noise in the same phonetic conditions is higher for [s] than for [sh], which is observed and with the normative pronunciation of these sounds.

Let us compare the obtained results of the study with the characteristics of other slotted consonants. To do this, we will compare the averaged data on the spectral characteristics of defective consonants in the experiment, obtained by averaging the frequency values for specific syllables ([sa], [so], [su], [sy], [as], [os], [us], [ys], [sha], [sho], [shu], [shi], [ash], [osh], [ush], [ysh]). These data were identified separately for each type of defect with averaged data on the frequency characteristics of the slotted sounds, which are close to defective sounds in their articulatory features. 
Interdental sigmatism. The English consonant [f] is the closest to this consonant. When these sounds are pronounced, the tip of the tongue is located between the upper and lower teeth; the tongue is flat and lightly tense. English [f] is flat-slit sound, unsharp, which makes it low-intensity with a brighter formant structure than that of circular slit sounds. The frequency characteristics of the interdental [s] and [sh] (upper and lower noise limits) are very close to the frequency characteristics of sound [f]. Interdental pronunciation is characterized by a lower noise floor (about $1450 \mathrm{~Hz}$ and $1475 \mathrm{~Hz}$ ) compared to standard sounds. Also, with this type of distortion, there is a large noise bandwidth, noise intensity, which corresponds with those signs that are noted with interdental articulation.

Labiodental sigmatism. Frequency characteristics with this variant of defective pronunciation bring labiodental [s] and [sh] closer to the English consonant [f]: the upper noise limit is about $8000-9000 \mathrm{~Hz}$, the lower one is about $1500-1600 \mathrm{~Hz}$. The noise spectrum is very smooth, low-intensity, which is typical for bilabial and labiodental articulations.

Lateral sigmatism. Lateral articulation is normal for the consonant [1], which has a spectral pattern similar to the pattern of vowels, but with antiresonance in the F2-F4 area, causing the presence of air space behind the edge of the tongue and additional amplification in the F4 area. It is also characterized by the absence of a change in frequency characteristics typical for other consonants in front of rounded vowels. These vowels cause a decrease in the lower border of the noise, because the acoustic effect of velarization is similar to the effect of labialization. A normal amplification in the range of $4-6 \mathrm{kHz}$ appears in closed syllables with lateral pronunciation, possibly similar to an additional resonance in the F4 area, which is characteristic of lateral consonants. Of all the listed signs, only the last one was typical for lateral sigmatism. The frequency characteristics of the defective [s] and [sh] with lateral pronunciation turned out to be as close as possible to the normative sounds in terms of the position of the upper and lower boundaries and, accordingly, the width of the noise band. This manifestation can be explained by the fact that, when pronouncing the consonant $[\mathrm{s}]$ in the lateral sigmatism, the front part of the back of the tongue remains lowered, the tip of the tongue is pressed against lower teeth, as with the standard [s], and with the sound [sh] - the front part of the back of the tongue is raised. This happens in contrast to other defects in contrast to other defects (interdental, dental, labiodental).As a result, only the place of the exhaled air stream changes (i.e., when pronouncing the sound [s], a central compression is formed; and when pronouncing [sh], the raised position of the tip and the front of the back of the tongue with a lateral slit in the mouth remains). Then how central air jet remains in other types of sigmatisms, but the size of the resonator changes by modifying the position of the front of the back and the tip of the tongue.

Dental sigmatism. The low-intensity noise and the presence of a significant number of acoustic zeros in the spectrum are characteristic for both forward and reverse syllables. Such characteristics can be explained by the position of the tongue during the dental pronunciation, which makes it difficult for the free passage of the air stream. The noise is lower than normal: from $2.5-3 \mathrm{KHz}$ to $6-7.5 \mathrm{KHz}$ for the consonant [s] and from 2.5-3.5 $\mathrm{KHz}$ to $7.5-8 \mathrm{KHz}$ for [sh]. In all cases, the upper and lower boundaries of the noise band for dental sigmatism are lower (except for the syllables [sy] and [shi], where the upper limit of the noise bandwidth correspondes with the normative). There are no similar consonants in the norm in terms of spectral characteristics. Dental pronunciation does not similar any position, both in terms of the mode of formation and the place of formation.

Labial sigmatism (for the consonant [sh]). High-intensity noise and an increase in areas of maximum energy concentration are observed in all syllables of different structures and with different vowels (especially $[\mathrm{o}]$ and $[\mathrm{u}]$ ). This manifestation can be explained by the fact that in this variant of defective pronunciation, the tongue does not take part in 
articulation, lies freely on the bottom of the oral cavity, and the sound is formed due to a strong, intense exhalation of an air stream through rounded lips. In all cases, the lower limit of the noise bandwidth for labial sigmatism is lower than the normative [sh]. This effect leads to an excessive increase in the noise bandwidth and, consequently, the noise intensity. The labial pronunciation of the consonant [sh] has no analogs in its spectral characteristics among other consonant sounds, as well as in the dental stigmatism.

The research materials show, that the lateral pronouncing voiceless fricative consonants of all types of defective pronunciation is the closest to the normative on their own spectral characteristics. Although when perceived by ear, it differs sharply from the existing acoustic standard. Interdental articulation, which has a weaker fricative noise and a longer spectrum, brings interdental sounds [s] and [sh] closer to English [f]; and the frequency characteristics of labiodental ones - with the Russian consonant [f]. Dental pronunciation of [s] and [sh] and the labial pronunciation of [sh] have no analogues in the normative pronunciation in terms of their spectral characteristics.

\section{Discussion}

Research data, which were obtained experimentally in the process of experimental-phonetic study, indicate the presence of general and specific acoustic characteristics of various types of defective sounds pronunciation in syllables with various structures.

There is an increase in noise intensity and a tendency to keep areas of maximum energy concentration in all variants of defective sounds pronunciation in the structure of a closed syllable (in comparison with the structure of an open one). This feature brings these sounds closer to the norm. The character of articulation ("articulatory continuity") affects the acoustic characteristics of sounds in different ways, when pronouncing different syllable structures. The data obtained by the experimental phonetic method should be taken into account when selecting speech material and the sequence of its presentation at the stage of sound automation.

\section{Conclusions}

1. Analysis of the spectrograms of defective pronunciation of the sounds [s] and [sh] in the structure of syllables consonant-vowel and vowel-consonant allows us to say that the consonant and the following vowel are the most strongly connected; the vowel and the following consonant are the most independent. This fact can be explained by the different nature of the articulatory influence of sounds, when pronouncing a syllable.

2. When reproducing the structure of the syllable consonant-vowel at the very beginning of the articulation of the consonant sound, the articulatory organs tend to take the position necessary for pronouncing the vowel. Such preliminary preparation for pronouncing a vowel leads to the fact that the consonant is pronounced with the articulation of the subsequent vowel partially superimposed on it, which causes a change in the acoustic characteristics of the consonant sound. In this case, the most significant change that characterizes the sounds in the structure of the consonant-vowel syllable is their change under the influence of the roughening of the subsequent vowel. There were differences in the spectral characteristics of consonants compared to those in combination with other vowels for all variants of defective sounds pronunciation in the structure of the consonant-vowel syllable in combination with the vowels [o] and [u]. There was reducing the lower border of the noise band, decreasing in the total intensity of noise components. The greatest changes in the spectral characteristics of sounds in the structure of the 
consonant-vowel syllable are determined by their pronunciation fusion in all variants of defective pronunciation as well as in the normative pronunciation.

3. When the structure of the vowel-consonant syllable is reproduced, the articulation of the preceding vowel does not end by the beginning of pronouncing the consonant sound, which leads to the appearance in spectrum of a consonant area containing low-frequency components. Changes of the consonants under the influence of the roundedness of the preceding vowel, do not affect the consonant as a whole, in contrast to the structure of the syllable consonant-vowel. These changes extend only to a small section of the consonant spectrum (about 30-40 ms long) which is adjacent to the rounded vowel.

4. Close connection of sounds in the structure of the minimum pronunciation unit - syllable - is reflected in their acoustic characteristics. When considering the mutual influences of sounds in the structure of syllables such as consonant-vowel and vowel-consonant, it was found that these mutual influences are different. In the structure of a syllable such as consonant-vowel, the consonant sound is more susceptible to the influence of the vowel, and in the structure of the syllable type vowel-consonant is less susceptible. This is noted in all variants of defective pronunciation of sounds as well as in the normative pronunciation. The roundness of the vowel most significantly affects on the articulatoryacoustic characteristics of consonants. The roundedness of a vowel in the structure of syllable of type consonant-vowel leads to a decrease in the frequency of noise components of consonants, and in the structure of a syllable of type vowel-consonant - to an increase.

\section{References}

1. L. Lopatina, Speech therapy work on the correction of mild dysarthria in preschool children (UMC «Dobriimir», Moscow, 2015).

2. H. Chandrashekar, V. Karjigi, N. Sreedevi, Ieee transactions on neural systems and $\begin{array}{llllll}\text { rehabilitation engineering } 12 & \mathbf{( 2 8 )}, \quad 2880-2889 & \text { (2020). doi: }\end{array}$ 10.1109/TNSRE.2020.3035392.

3. S. Aradi, B. Cucchiara, Decision-making in adult neurology, 48-49. Elsevier, St. Louis, Missouri (2021).

4. J. Ogar, H. Slama, N. Dronkers, S. Amici, M.-L. Gorno-Tempini, Neurocase 6 (11), 427-432 (2005).doi: 10.1080/13554790500263529.

5. W. Ziegler, I. Aichert, A. Staiger, Journal of Phonetics 64, 145-155 (2017). doi: 10.1016/j.wocn.2016.10.002.

6. J. Lee, M.-A. Littlejohn, Z. Simmons, International Journal of Speech-Language Pathology 2 (19), 195-204 (2017). doi10.1080/17549507.2016.1193899.

7. K. Myungjong, K. Hoirin, Miesenberger, K. (eds.): ICCHP 2012, Part II, LNCS 7383, 447-450 (2012). Springer, Heidelberg. doi: 10.1007/978-3-642-31534-3_66.

8. P. Wannberg, E. Schalling, L. Hartelius, Logopedics. Phoniatrics. Vocology 4 (41), 159-167 (2016). doi: 10.3109/14015439.2015.1069889.

9. J. Whitfield, A. Goberman, International Journal of Speech-Language Pathology 2 (19), 184-194 (2017). doi: 10.1080/17549507.2016.1193897.

10. W. Ziegler, Cognitive Neuropsychology 7-8(34), 482-487 (2017). doi: 10.1080/02643294.2017.1421148.

11. L. Bulanin, Phonetics of modern Russian language (Librocom, Moscow, 2011).

12. B. Galantucci, C. Fowler, M. Turvey, Psychonomic Bulletin \& Review 13 (3), 361377 (2006). doi:10.3758/BF03193857 
13. E. Baković, Language, Cognition and Neuroscience, 1 (29), 21-23 (2014). doi: 10.1080/01690965.2013.848992

14. G. Lenoci, C. Celata, I. Ricci, Clinical linguistics \& phonetics. URL: https://www.tandfonline.com/doi/full/10.1080/02699206.2020.1853811. doi: 10.1080/02699206.2020.1853811 (2020).

15. J. Fritsch, M. Magimai-Doss, Ieee signal processing letters 28, 224-228 (2021). doi: 10.1109/LSP.2021.3050362

16. G. Van Nuffelen, C. Middag, M. De Bodt, J.-P. Martens, International Journal of Language \& Communication Disorders 5 (44), 716-730 (2009). doi: $10.1080 / 13682820802342062$

17. L. Baryaeva, T. Volosovec, O. Gavrilushkina, Sample adapted basic educational program for preschoolers with severe speech disorders (2ndedn. CDK prof. L.Baryaevoi, Moscow, 2015)

18. E. Levy, International Journal of Speech-Language Pathology 4 (16), 344-354 (2014). doi: $10.3109 / 17549507.2014 .894123$ 